

\section{Forward Physics with Rapidity Gaps at the LHC}

\section{M.G.Albrow ${ }^{a *}$, A.DeRoeck ${ }^{b}$, V.A.Khoze ${ }^{c}$, J.Lämsä $^{d, e}$, E.Norbeck $^{f}$, Y.Onel $^{f}$, R.Orava $^{e}$, and M.G.Ryskin ${ }^{g}$.}

${ }^{a}$ Fermi National Accelerator Laboratory, Batavia, IL60510, U.S.A.

${ }^{b}$ CERN, CH-1211 Geneva, Switzerland.

${ }^{c}$ IPPP, Dept. of Physics, Durham University, Durham, U.K.

${ }^{d}$ Iowa State University, Ames, Iowa, U.S.A.

${ }^{e}$ Dept. of Physics, Univ. of Helsinki, and Helsinki Inst. of Physics, Finland.

${ }^{f}$ Univ. of Iowa, Iowa City, Iowa, U.S.A.

${ }^{g}$ Petersburg Nuclear Physics Inst., Gatchina, St.Petersburg, Russia.

E-mail: albrow@fnal.gov, orava@mail.cern.ch

ABSTRACT: A rapidity gap program with great potential can be realised at the Large Hadron Collider, LHC, by adding a few simple forward shower counters (FSCs) along the beam line on both sides of the main central detectors, such as CMS. Measurements of single diffractive cross sections down to the lowest masses can be made with an efficient level-1 trigger. Exceptionally, the detectors also make feasible the study of Central Diffractive Excitation, and in particular the reaction $g+g \rightarrow g+g$, in the color singlet channel, effectively using the LHC as a gluon-gluon collider.

KEYWORDS: Instrumentation for particle accelerators and storage rings - high energy; Trigger detectors; Scintillators.

\footnotetext{
${ }^{*}$ Corresponding author.
} 


\section{Contents}

1. Introduction 1

2. Single Diffractive Excitation

3. Central Exclusive Production (CEP) 5

4. Efficiency for detecting rapidity gaps and for rejecting background 8

5. Single particle efficiency of FSCs 9

6. Single diffraction detection efficiency 10

7. Central exclusive production detection efficiency 13

8. Non-diffractive detection efficiency 15

9. Conclusions 15

10. Acknowledgements 15

\section{Introduction}

The principal goal of the program of forward physics at the Large Hadron Collider, LHC, is the measurement of the main characteristics of diffractive interactions. These processes are very significant in their own right, to better understand QCD in the non-perturbative regime, and they form a large fraction of the total cross section. In addition, they are valuable because of their intimate connection to the rapidity gap survival factor $\hat{S}^{2}$, which determines the rate of suppression of the central exclusive processes caused by rescattering effects (including additional parton interactions). Such processes have a number of unique advantages for studying QCD and new physics, and in particular for detailed probing of the Higgs boson properties [1], 凤]. 


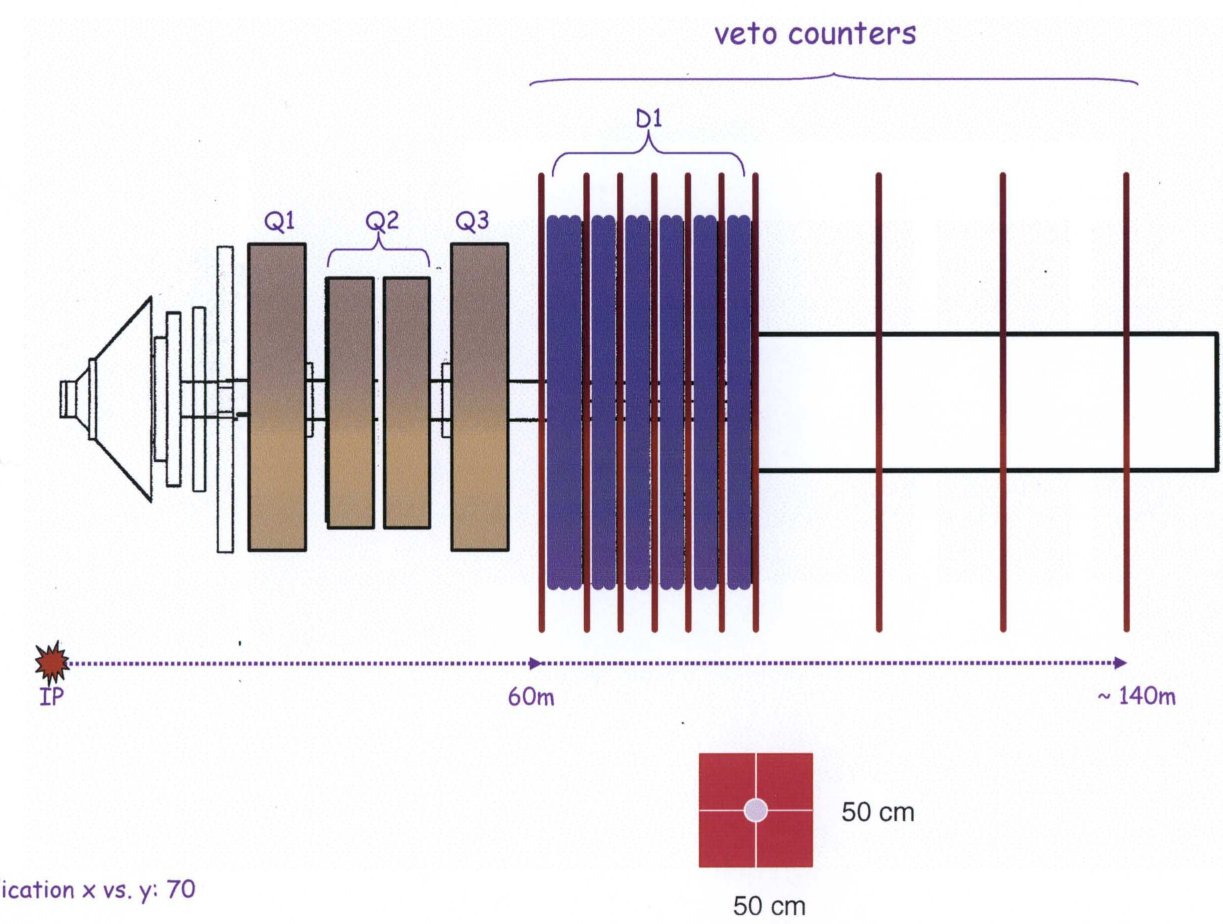

Figure 1. The proposed layout of the FSC counters on both sides of the CMS intersection region from $z= \pm 60 \mathrm{~m}$ to $z= \pm 140 \mathrm{~m}$. The vertical lines indicate the locations of the proposed counters.

For predictions of soft diffractive processes at the LHC we need a reliable theoretical model. There has not been much improvement in the theoretical understanding of the high energy behaviour of the strong interaction amplitude since the late 1960s [3]. For recent reviews, see Refs [4, 5]. For instance, different asymptopic behaviours of such a fundamental quantity as the total $p p$ interaction cross section, $\sigma_{T}$, are not excluded. Thus in the "weak coupling" regime, $\sigma_{T}(s \rightarrow \infty) \rightarrow K$, where $K$ is a constant, while in the "strong coupling" regime $\sigma_{T} \sim \ln (\mathrm{s})^{\varepsilon}$ with $0<\varepsilon<2$. Note that for these two regimes different behaviour of the cross section for diffractive dissociation is predicted. It is therefore important to study different channels of diffractive dissociation at the LHC to make progress.

The experimental results available at present are fragmentary, and do not cover the whole kinematic range. To further constrain the parameters of the models of soft diffraction it is crucial to make accurate measurements at LHC energies of the single diffractive dissociation cross section for low masses, $\sigma_{S D}\left(\right.$ low $M$ ), and of central diffractive production, $\frac{d \sigma}{d \eta_{1} d \eta_{2}}$, where $\eta_{1}$ and $\eta_{2}$ define the pseudorapidity $\left(\eta=-\ln \tan \frac{\theta}{2}\right)$ range of the central system.

While the physics of diffractive dissociation at the LHC is important, the existing LHC detectors (ALICE, ATLAS, CMS, and LHCb) are not well suited, as they lack the coverage necessary to measure forward rapidity gaps. The TOTEM experiment [6, 7] will measure the total cross section and elastic scattering, detecting protons in Roman pots, and make some studies of high cross section diffractive interactions. Downstream of the ATLAS experiment, the ALFA project consists of a set of Roman pots with fiber tracking, also for elastic scattering and total cross section measurements. In this paper, the concept of using forward shower counters, FSC, to detect and trigger 


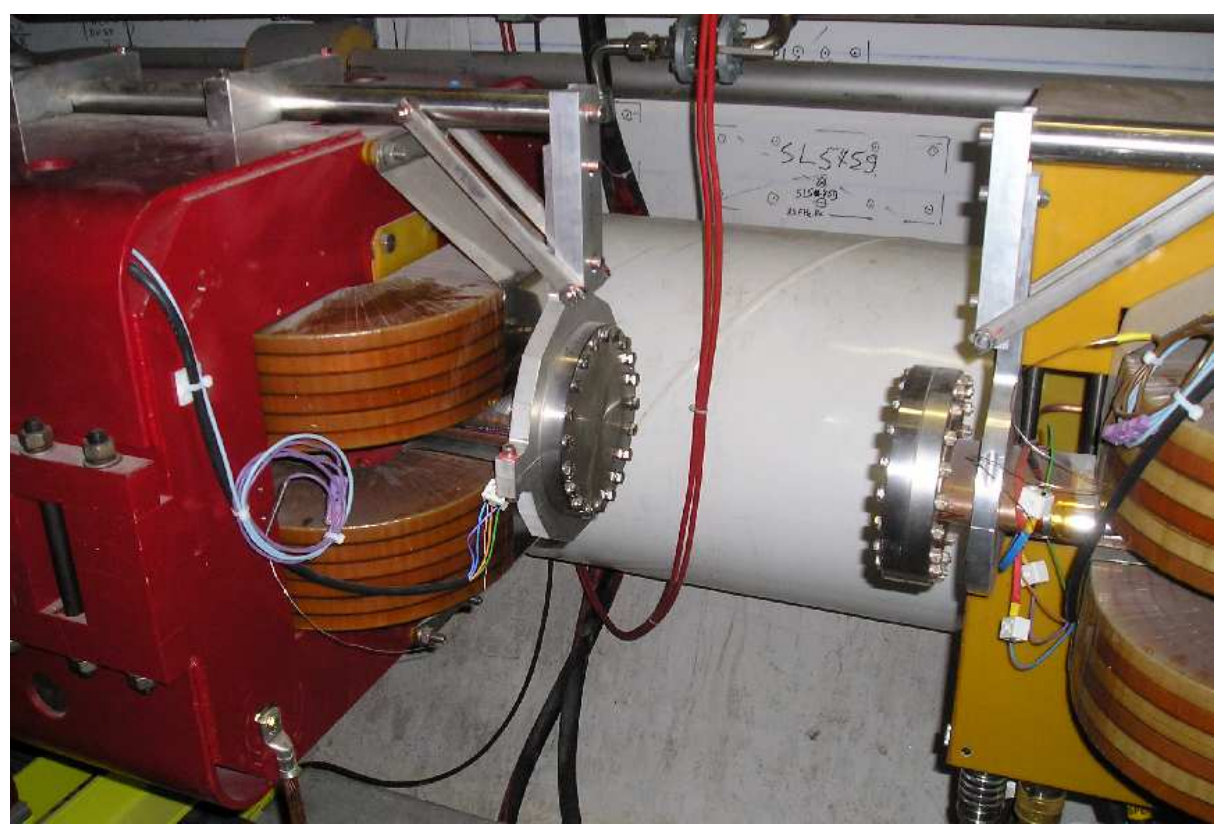

Figure 2. Region between the MBXW magnets where the FSC counters can be installed. (Photograph taken before the connecting pipe was installed; it shows accessible elliptical beam pipe regions.)

on rapidity gaps in diffractive events at the LHC is presented. The benefits of the FSC system for the study of single and central diffractive reactions are shown. Extensive simulations of several reactions have been performed to establish the efficiency of the FSC detector arrangement. The main application of these studies will be for low luminosity running with a small probability of pile-up (more than one inelastic $p p$ collision per bunch crossing). This study parallels that of Ref. [8] and that presented in a proposal to the CMS Collaboration [9]. In this paper we refer specifically to CMS, but the other three central detectors may similarly benefit from such very forward detectors.

Scintillation counters can be added closely surrounding the beam pipes, with $60 \mathrm{~m}<|z|<$ $85 \mathrm{~m}(\mathrm{z}$ is the coordinate along the beam direction), and at further locations out to $z= \pm 140 \mathrm{~m}$ on both sides of the interaction point (IP5), see Figure 1. At these locations the beam pipes are accessible, and not in a cryogenic region. An option is to supplement scintillation counters with gaseous electron multipliers (GEMs), which would add spatial segmentation, and therefore shower position information. The FSC do not detect particles directly from the collisions, but they detect showers from forward particles that interact in the beam pipe and surrounding material. These detectors can be used to make measurements of rapidity gaps for events without pile-up, in the early days of LHC running. The average number of inelastic collisions per bunch crossing, $\langle n\rangle$, for $\sigma_{\text {inel }}$ $=80 \mathrm{mb}, 6 \times 10^{11}$ protons per bunch, 156 bunches and $\beta^{*}=2 \mathrm{~m}$ is $\langle n\rangle \sim 1.0$, and then $e^{-\langle n\rangle}=0.368$ is the fraction of bunch crossings with no inelastic collisions (and $36.8 \%$ of diffractive, or any other specific, collisions are not spoiled by pile-up). These conditions give the highest rate of diffractive collisions without pile-up. When $\langle n\rangle=2(3)$ rapidity gap physics can still be done, but with lower 
efficiency, $\varepsilon=13.5(5.0) \%$ respectively. As most of the pile-up events will have forward particles giving showers in the FSC, they can be effectively vetoed at the level-1 trigger, increasing the efficiency of diffractive triggers. For single diffractive excitation one would require all the counters on one side (in logical OR) to be consistent with noise. Showers will usually give a large pulse height, many times that of a minimum ionizing particle (MIP), and are easily discriminated from noise. Off-line, multiple events in a bunch crossing also usually give more than one primary vertex, as reconstructed from the excellent tracking capabilities of CMS. However low mass diffractive excitation can have all particles at small polar angles, and in some cases will not have measured tracks, or they will not form a reconstructable primary vertex.

For simplicity of discussion, consider level-1 triggers in configurations of gaps (detectors consistent with noise) or particles (signals significantly above noise), in three rapidity regions: forward "East" side, central, and forward "West" side. "Central" may e.g. mean some specified activity in $|\eta|<3$. Call [000] all empty, [001] particles only forward(West), etc., thus eight possible configurations. Uninteresting crossings with no inelastic interaction are [000], and non-diffractive and pile-up events are [111]; these will dominate zero-bias data and are not relevant for diffractive physics. It is interesting to trigger on [001]+[100], representing low-mass single diffraction, [011]+[110] which is high-mass single diffraction, [101] which is double diffractive dissociation, and [010] which is double pomeron exchange (central diffraction). These six triggers all involve large ( $\gtrsim 5$ ) rapidity gaps and will contain no pile-up events, so their rate will decrease approximately exponentially once $\langle n\rangle \gtrsim 3$.

In addition to their value in triggering on and studying diffractive collisions without pileup, the FSC counters can provide real-time monitoring of beam conditions (beam halo) for both incoming and outgoing beams (which are both in the same pipe at these locations). The separation of incoming and outgoing beams can be done by timing the scintillation counter signals at a few locations where their time separation is $\gtrsim 10 \mathrm{~ns}$ (the maximum being $12.5 \mathrm{~ns}$ ). This is likely to be a useful beam diagnostic, especially in early LHC operation.

These detectors will allow the measurement of low mass single diffractive dissociation, $p+p \rightarrow p+p^{*} \rightarrow p+X$, where $X$ is a system of particles with typically $M(X) \sim$ few GeV. This physics is not possible with the central detectors, as the hadrons coming from the fragmentation of $X$ typically have forward (longitudinal) momenta $\sim \mathrm{TeV} / \mathrm{c}$ and transverse momenta $p_{T} \lesssim 1 \mathrm{GeV} / \mathrm{c}$. The FSC can not reconstruct the forward primary hadrons, but the patterns of their signals can be compared with simulations of soft diffraction to test the models. Such data will strongly constrain existing models of diffractive processes and the effects of rescattering and spectator parton interactions, see for instance Ref. [10]. This information is needed in order to understand the rapidity gap dynamics and gap survival probability. No special running is needed; the standard low- $\beta$ running can be used, but most effectively with low luminosity per bunch crossing such that $\langle n\rangle \lesssim 3$. With 25 ns bunch spacing (2808 bunches), for $\sigma_{\text {inel }}=80 \mathrm{mb}$, this means luminosity $L<4 \times 10^{32} \mathrm{~cm}^{-2} \mathrm{~s}^{-1}$. Even when a store starts at $L=10^{33} \mathrm{~cm}^{-2} \mathrm{~s}^{-1}$, after a few hours this condition will probably be met.

The purity of the diffractive data sample (and hence the statistics for a given trigger band- 
width) will be higher with one FSC arm in veto. The Collider Detector at Fermilab, CDF, installed a similar set of counters (beam shower counters, BSC) used in veto at level-1 for some physics (central exclusive production) [11]. These were pairs of scintillation counters, closely surrounding the beam pipe, at each of three (four on one side) locations, $6.6 \mathrm{~m}, 23.2 \mathrm{~m}, 31.6 \mathrm{~m}$ (and $56.4 \mathrm{~m}$ ) from the intersection point. The closest counters had acceptance for primary particles with $5.4<|\eta|<5.9$, and were preceded by two radiation lengths of lead to convert photons. The other counters were behind quadrupoles, electrostatic separators and (for the last counter) a dipole magnet. These only detected showers produced by particles in the beam pipe and surrounding material. Together they covered $5.4<|\eta|<7.4$. The Tevatron beam has $y(p)=7.65$, where rapidity $y(p)=\ln \frac{\sqrt{s}}{m(p)}$. They were used effectively both in level-1 triggers, and to tag events with proton dissociation. CDF also had a set of Roman pot detectors, with tracking, to measure diffractively scattered antiprotons. It was found that even when $\langle n\rangle<1$, high mass single diffraction studies ( $X=$ dijets, $W, Z+$ hadrons) with a $\bar{p}$ track are dominated by pile-up (the $\bar{p}$ and $X$ being from different collisions) unless a rapidity gap in the $\bar{p}$ direction is required to veto pile-up. Note however that in central exclusive production with both protons detected $(p+p \rightarrow p+X+p)$ 4-momentum conservation and precision (relative) timing of the protons enable physics to be done even with $\langle n\rangle \gtrsim 20$ [纤].

In the following, a physics motivation is given for single diffractive excitation studies, and especially for central exclusive production. We then present efficiency calculations.

\section{Single Diffractive Excitation}

Single diffractive excitation, SDE, is the process $p+p \rightarrow p+X$, where " + " represents a large ( $\gtrsim$ 3 units) rapidity gap, meaning no hadrons in pseudorapidity $\eta=-\ln \tan \frac{\theta}{2}$ between the outgoing

proton and the diffractive system $X$. (Strictly, true rapidity $y=\frac{1}{2} \ln \frac{E+p_{z}}{E-p_{z}}$ should be used, but for practical reasons $\eta$ is usually considered to be an acceptable approximation.)

The FSCs cover a crucial rapidity region between the zero degree calorimeters [12], ZDC, in CMS and ATLAS (which only detect neutrons and photons produced close to $\theta=0^{\circ}$ ), CASTOR [13], and the TOTEM detectors T2 [6]. The dependence of $M(X)$ on rapidity gap size $\Delta \eta$ is $\left(\frac{M(X)}{\sqrt{s}}\right)^{2} \sim e^{-\Delta \eta}$, which can be used to estimate the mass spectrum, after correcting for the more detailed relationship using Monte Carlo expectations. Note that the true rapidity of the diffractively scattered proton is $y \sim \ln \frac{\sqrt{s}}{m(p)}=9.3$ (9.6) at $\sqrt{s}=10$ (14) TeV, but in the forward region $\eta$ and $y$ can be very different. A diffractively scattered proton with $p_{T}=0.25(0.5) \mathrm{GeV} / \mathrm{c}$ has $\eta(p)=10.9$ (10.2) at $\sqrt{s}=14 \mathrm{TeV}$ (and 3\% less at $\sqrt{s}=10 \mathrm{TeV}$ ). A particle with $p_{T}=0, \theta=0$ has $\eta=\infty$.

\section{Central Exclusive Production (CEP)}

In the following the reaction $p+p \rightarrow p+X+p$, where $X$ is fully measured and no other parti- 


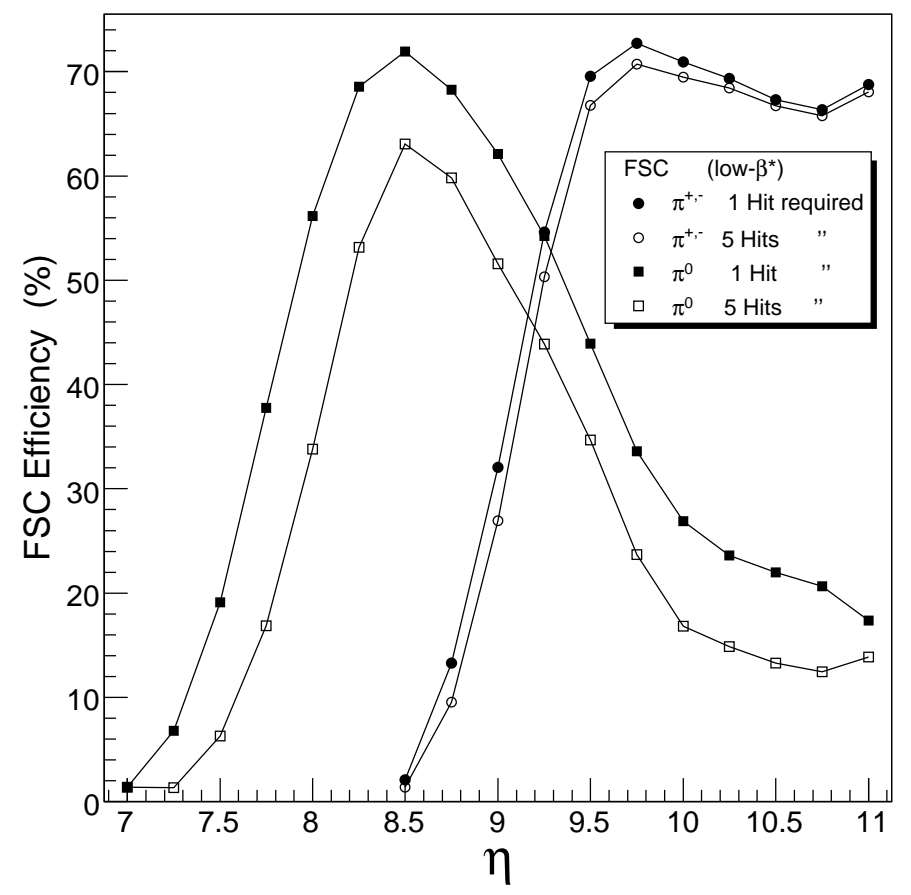

Figure 3. The efficiency (\%) of the forward shower counters (FSC) for registering particle showers induced by primary $\pi^{ \pm}$and $\pi^{0}$ as a function of their pseudorapidity $\eta$ (low $\beta^{*}$ conditions).

cles are produced, is studied. We call this central exclusive production, CEP, or central exclusive diffraction, CED. The FSCs are used in the definition of rapidity gaps (denoted + ), where a gap means no hadrons. In this study, with no pile-up, proton tagging is not required. This is quite different from the FP420 proposal [2], which is based on a precision measurement of both forward protons. This reaction allows for a rich physics program [10], and in particular it provides a unique possibility to study the two-gluon mediated color singlet interaction (i.e. using the LHC as a "gluongluon collider" $g g \rightarrow g g$ ). In central exclusive production (or diffraction) the $g g \rightarrow q \bar{q}$ background is, in general, suppressed compared to $g g \rightarrow g g$ due to color and spin factors. The selection rule $J_{z}=0$ [14], valid for the CEP processes, further suppresses this background by a factor $\propto\left(\frac{m(q)}{E_{T}}\right)^{2}$, where $m(q)$ is the quark mass and $E_{T}$ is the transverse energy of the jet. By excluding inclusive central diffraction with relatively large proton transverse momenta $\left(p_{T} \gtrsim 0.7 \mathrm{GeV} / \mathrm{c}\right)$, effective "gluon collider" conditions are reached [10]. As a result, detailed studies of pure high energy gluon jets can be made. To guarantee the purity, it is essential to reject central inclusive diffraction (CID) background, where one or both protons dissociates into a multiparticle state, or the jets are accompanied by unassociated particles. These are "direct backgrounds", not a pile-up effect. The FSC would effectively veto the dissociation background, as most of the fragmentation products hit the beam pipes and make showers, which are detected.

The physics program includes a search for the production of mesonic states, such as glueballs, hybrids, heavy quarkonia $\chi_{c}, \chi_{b}$ in double pomeron, $\mathbb{P} \mathbb{I P}$, reactions as well as photoproduc- 
tion: $\gamma \mathbb{P} \rightarrow J / \psi, \psi(2 S), \Upsilon(\mathbb{P}$ denotes the pomeron). A strong coupling for the reaction $g g \rightarrow M$ for mesons $M$ is expected as a result of two gluon exchange. A rapidity gap trigger on both arms could be used for this study. The quantum numbers of the central state are restricted: the process is a "quantum number filter". The $t$-channel exchanges over the large ( $\Delta y \gtrsim 5$ units) rapidity gaps can only be color singlets with charge $Q=0$ and spin $J$ (or effective spin $\alpha(t)) \geq 1$. Known exchanges are the photon $\gamma$ and the pomeron $\mathbb{P}$. The gluon satisfies the $J=1, Q=0$ requirements, but it is not a color singlet; however one or more additional gluons can cancel its color and form a pomeron. Another possible, but not yet observed, exchange in QCD is the odderon, $O$, a negative C-parity partner to the $\mathbb{P}$ consisting of at least three gluons. This physics program includes sensitivity to odderon exchange. States with $J^{P C}=1^{--}$such as the vector mesons $(V) J / \psi$ and $\Upsilon$ are produced primarily by photoproduction, $\gamma \mathbb{P} \rightarrow V$. They can also be produced by odderon exchange, $O I P \rightarrow V$, the signature for which would be a higher cross section for exclusive vector meson production than that expected from photoproduction (and as predicted from HERA data, where the $O$ is absent), and with vector mesons having higher $p_{T}$ (on average). This is because on average $p_{T}(O) \gtrsim p_{T}(\mathbb{P}) \gg p_{T}(\gamma)$.

There is a long list of hadronic states which can be studied, profiting from the quantum number filter. Glueball, $G$, spectroscopy, perhaps especially in the $G \rightarrow \phi \phi$ channel, and the exclusive production of hyperon pairs, e.g. IPIP $\rightarrow \Lambda \bar{\Lambda}, \Sigma \bar{\Sigma}$ are examples out of many that have not yet been studied by this technique. Many studies were done at the SPS (fixed target), but the energy was too low for $\mathbb{P} \mathbb{P}$ dominance. (One can not have two rapidity gaps $\Delta y \gtrsim 3$ units, with (say) 1 central unit for particle production, unless $\sqrt{s}>M(p) \times e^{3.5}=31 \mathrm{GeV}$, i.e. $p_{\text {beam }}=546 \mathrm{GeV} / \mathrm{c}$.) The highest $\sqrt{s}$ at which the exclusive hadronic processes such as $\mathbb{P} I P \rightarrow \pi^{+} \pi^{-}, K^{+} K^{-}, p \bar{p}$ and $\pi^{+} \pi^{-} \pi^{+} \pi^{-}$ were studied was $\sqrt{s}=63 \mathrm{GeV}$ [15] at the CERN ISR. (Actually $\alpha \alpha \rightarrow \alpha+\pi^{+} \pi^{-}+\alpha$ was measured at $\sqrt{s}=126 \mathrm{GeV}$, but the $\alpha$-particles had the same rapidity as the $p$. Indeed the $M\left(\pi^{+} \pi^{-}\right)$ spectrum was the same as in $p p$ collisions at $\sqrt{s}=63 \mathrm{GeV}$.) The $\pi^{+} \pi^{-}$mass spectrum showed striking structures: a probable $\sigma(600)$ broad enhancement, a narrow $f_{0}(980)$ and a clear dip around $1.6 \mathrm{GeV} / \mathrm{c}$, which has still not been fully understood but may be a manifestation of a glueball state.

A valuable reaction for calibrating forward spectrometers, e.g. as in FP420 [2], is the production of exclusive di-leptons from $\gamma \gamma$ interactions and from $\Upsilon$ decay. A level-1 trigger, based on two muons or two electromagnetic (EM) clusters and vetoing on the FSC counters, ZDC, and (in CMS) T1 and T2 detectors and the HF calorimeters, will select interactions with very large rapidity gaps and no pile-up. The rate of such events will be acceptable even with a low $(\sim 4 \mathrm{GeV}) E_{T}$ cluster threshold and a similar low- $p_{T}$ threshold for muons, thus including $\Upsilon \rightarrow e^{+} e^{-}, \mu^{+} \mu^{-}$(for low $p_{T} \Upsilon$ ). The CDF observations of exclusive lepton pairs and charmonium states [11] have been made possible thanks to their beam shower counters (BSCs).

More generally, properties of central systems that have not previously been measured will be examined. In addition to low mass exclusive central states (with all particles reconstructed, and mass $\left.M(X) \lesssim 10 \mathrm{GeV} / \mathrm{c}^{2}\right)$, the same trigger will collect high mass double pomeron events, with $M(X)$ up to about $100 \mathrm{GeV} / \mathrm{c}^{2}$. The jet content of such events, and in particular the subset of exclusive di-jets, is a valuable probe of the parton constituents in the pomeron. Most such jets should 


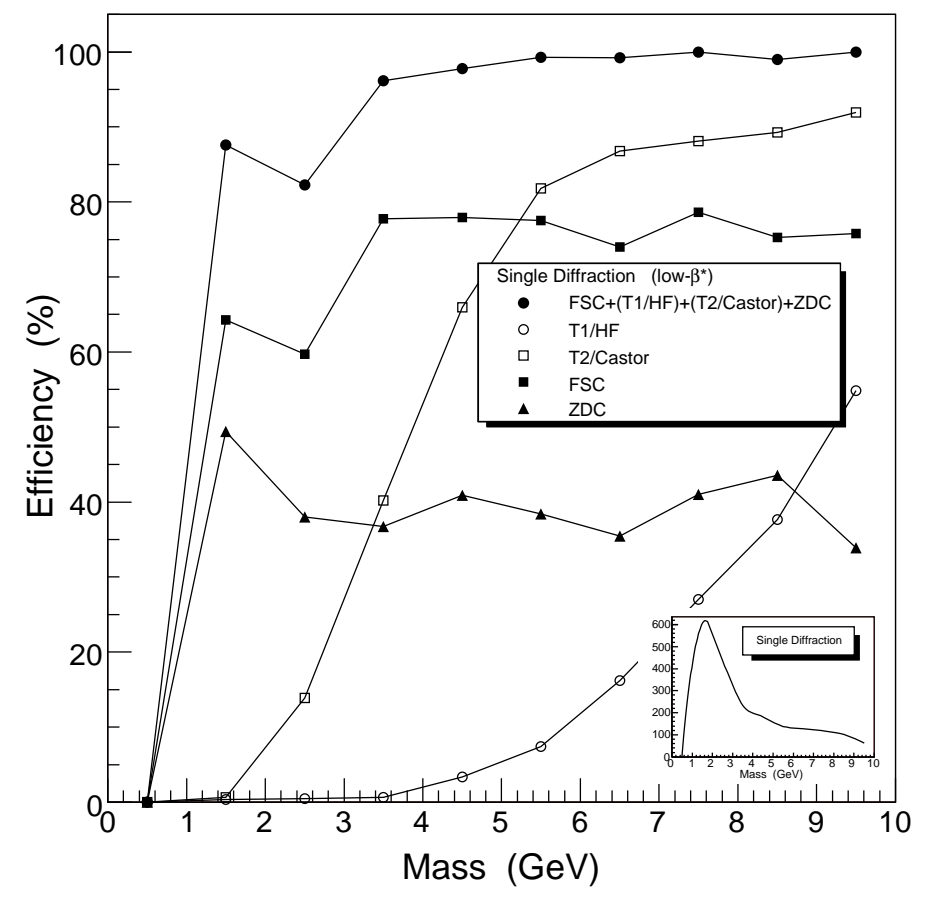

Figure 4. The detection efficiencies for single diffrative events simulated by PYTHIA6.2 as a function of the diffrative mass. We required at least five charged particles ("hits") in any of the forward shower counters, or at least one track in the $\eta$ region covered by T1/HF or T2/CASTOR, or a minimum energy deposit in the ZDC (see text).

be gluon jets. One can compare $\mathbb{P}+\mathbb{P}$ collisions at $M(X)$ with $p p$ collisions at $\sqrt{s}=M(X)$. Some differences may be: a larger content of $\eta$ and $\eta^{\prime}$ mesons and a smaller baryon fraction because of the higher glue content. Pomerons should have a smaller transverse size than protons, and this may manifest itself in relatively more double parton scattering $(2 \times(g g \rightarrow J J))$ and perhaps also in BoseEinstein correlations, which measure the size of the pion emission region. Double parton scattering, seen in 4-jet events having two pairwise-balancing dijets, is a probe of the (unintegrated) two-gluon density $G_{2}\left(x_{i}, x_{j}\right)$. Other ideas for early LHC running using forward detectors for rapidity gap based physics have been presented [10]. Furthermore the proposed FSCs would provide information of value to the FP420 initiative [2] to place leading proton detectors at $\pm 240 \mathrm{~m}$ and $\pm 420 \mathrm{~m}$ from intersection Points 1 (ATLAS) and 5 (CMS).

\section{Efficiency for detecting rapidity gaps and for rejecting background}

The FSC locations are in front of and behind the separation dipole D1, and also between each of the six MBXW elements of D1 (see Figure 1). In addition counters can be placed 15 m, 35 m, and $55 \mathrm{~m}$ beyond D1, making a total of 10 detectors on each side. We have included in our efficiency calulations the T1, T2, HF, CASTOR (one side only) and ZDC detectors. The TOTEM tracker T1 and the forward calorimeter HF span the region $3<|\eta|<5$. Tracker T2 and the CASTOR 


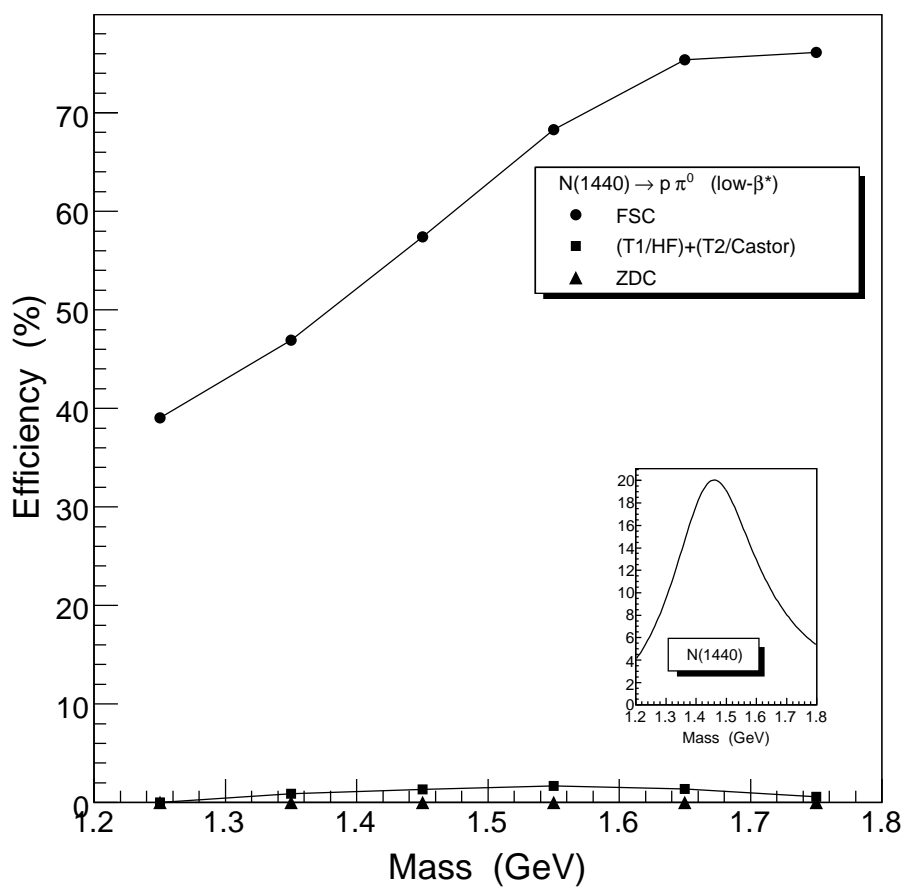

Figure 5. The detection efficiency for single diffractive events with $N^{*} \rightarrow p+\pi^{0}$ as a function of diffractive mass. We required at least five hits in any of the forward shower counters, or at least one track in the $\eta$ region covered by T1/HF or T2/CASTOR, or a minimum energy deposit in the ZDC (see text).

calorimeter cover $5<|\eta|<7$. The Zero Degree Calorimeter, ZDC, is in-between the two beam pipes just beyond their separation, and detects only neutral particles (mainly $\gamma$ and neutrons) with $|\eta|>8.5$. The program GEANT [16] has been used to simulate the beam line, including the beam pipes, beam screens, and magnetic elements. The running condition is for the standard low- $\beta$ configuration, $\beta^{*}=0.55 \mathrm{~m}$; no special running is required for this program. However its efficiency will become less than $5 \%$ when the average number of inelastic collisions per bunch crossing, $\langle n\rangle>3$.

\section{Single particle efficiency of FSCs}

The FSC detection efficiency for incident particles $\left(\pi^{ \pm}, \pi^{0}\right)$ was calculated as a function of pseudorapidity $\eta$. The requirement was at least one particle ("hit") (alternatively at least five) in any of the FSC counters. A transverse momentum $\left(p_{T}\right)$ distribution of the form $e^{-6.7 p_{T}^{2}} \cdot d p_{T}^{2}$ was assumed for the incident primary particles, corresponding to that obtained from PYTHIA 6.2 [17]. The efficiency of the FSCs for detecting charged particles from showers induced by the primary $\pi^{ \pm}$and $\pi^{0}$ is shown in Figure 3. For charged pions the efficiency is $\sim 70 \%$ for $|\eta|>9.5$, and it is nearly independent of the number of hits, at least for $1-5$ hits per detector plane. For $\pi^{0}$ between 8 $<|\eta|<9.3$ it exceeds 65\% (50\%) when at least 1 (5) hits are required. From the results presented in the following sections, this is sufficient for the anticipated physics studies. 


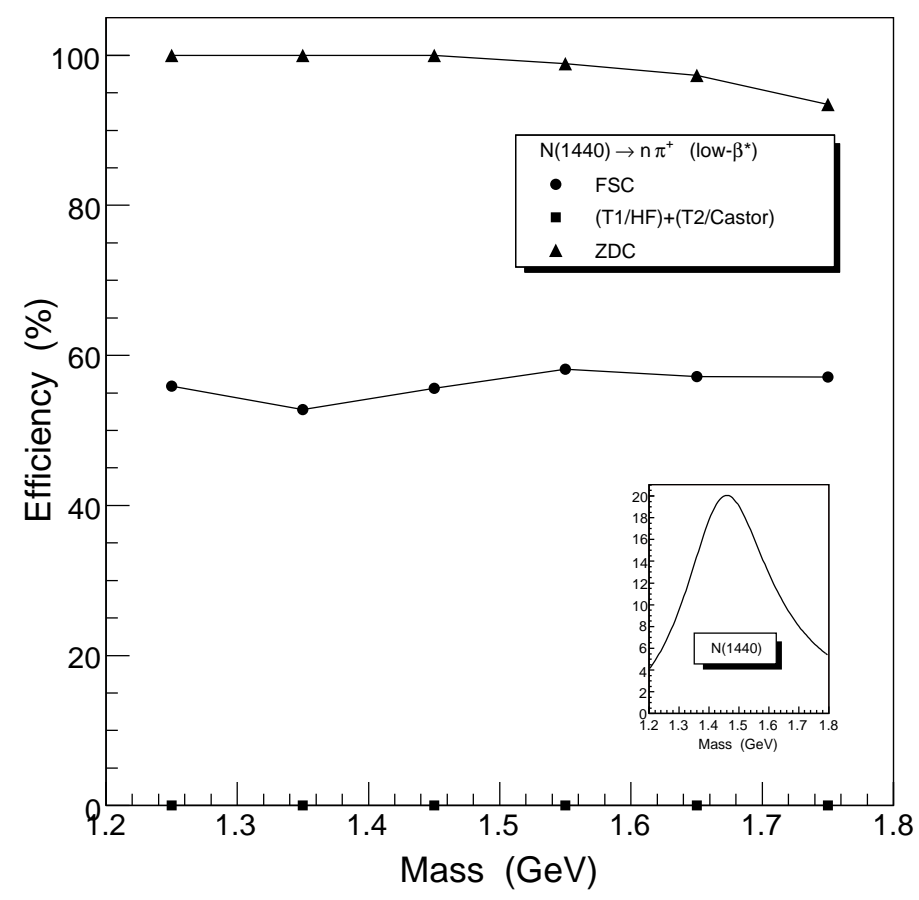

Figure 6. As Fig.5 for $N^{*} \rightarrow n+\pi^{+}$.

\section{Single diffraction detection efficiency}

The detection efficiencies for single diffractive excitation, as simulated with PYTHIA 6.2, were calculated as a function of the diffractive mass. They were also calculated with PHOJET 6.2 [18] and found to agree with those from PYTHIA, within statistics. We required at least five hits in any FSC counter, or a track or signal in the $|\eta|$ region covered by T1, T2, HF, CASTOR or the ZDC. A "signal" in the HF or CASTOR is defined as an energy deposit above $15 \mathrm{GeV}$, or above $500 \mathrm{GeV}$ in the ZDC. The $500 \mathrm{GeV}$ is nominal. Once data are obtained at low luminosity with a zero-bias (bunch crossing) trigger, it will be possible to optimise the cuts, for each detector, to provide the best separation between events with a true gap (no particles) and with particles. As in the CDF analysis, one can divide the zero-bias events into two classes: those apparently empty (no tracks and no large electromagnetic clusters) and those with interactions. For such studies it is necessary to have zero-bias data recorded, especially at low luminosity when the fraction of empty crossings is not too small. The efficiencies as a function of diffractive mass for these conditions, along with what would be obtained using only T1/HF, T2/CASTOR, FSC or ZDC detectors are shown in Figure 4. The efficiency is $>90 \%$ for the lower mass region, and approximately $100 \%$ for masses above 10 $\mathrm{GeV}$ (not shown). Approximately $25 \%$ of the single diffractive cross section is for masses below $10 \mathrm{GeV}$ (at $\sqrt{s}=14 \mathrm{TeV}$ ). Forward multiparticle states from central inclusive diffraction reactions would have similar detection eficiency.

Simulations have also been made for exclusive diffractive baryon resonance production, such as $p+p \rightarrow p+N^{*}(1440)$ with $N^{*} \rightarrow p+\pi^{0}, n+\pi^{+}$, or $\Delta^{++}+\pi^{-}$. In Figures 5-7 the 
efficiencies for detecting these final states are shown as functions of the diffractive mass. For $N^{*} \rightarrow p+\pi^{0}$ the average eficiency is $70 \%$ (Figure 5), for $N^{*} \rightarrow n+\pi^{+}$it is close to $100 \%$ (Figure 6 ), and for $N^{*} \rightarrow \Delta^{++}+\pi^{-}$it is about $70 \%$ (Figure 7).

An approximate calculation of the diffractive mass can be made through its relation to the size of the rapidity gap adjacent to the scattered proton, although this has some model dependence. The relation depends on the $p_{T}$ distribution (and hence $\left\langle p_{T}\right\rangle$ ) of the produced particles. The "adjacent rapidity gap" is defined as the gap between the diffractive proton (close to the beam rapidity, $y_{\text {beam }}=9.6$ at $\sqrt{s}=14 \mathrm{TeV}$ ) and the nearest particle in rapidity. Larger rapidity gaps correspond to smaller diffractive masses. The approximate correspondence between the diffractive mass $M$ and the (pseudo)rapidity gap $\Delta \eta$ is $\left(\frac{M(X)}{\sqrt{s}}\right)^{2} \sim e^{-\Delta \eta}$. From the theoretical point of view it is more instructive to consider the distribution $\frac{d \sigma_{S D}}{d \eta^{\prime}}$, where $\eta^{\prime}$ is the position of the edge of the gap. Although asymptotically $\frac{d M^{2}}{M^{2}}=d \eta^{\prime}$ due to angular ordering of gluon emission, different contributions to $\sigma_{S D}$ are separated from each other in $\eta^{\prime}$ rather than in $M$.

To provide a more precise (although model dependent) measurement, the PYTHIA program has been used to determine the correlation between the diffractive mass and the size of the rapidity gap. Figure 8 shows the true diffractive mass $M$ versus $\Delta \eta$ as determined by this method. To account for the measurement resolution, a Gausssian spread with $\sigma=10 \%$ has been added to the actual rapidity value. This is more than one unit at the largest values considered, and is considered to be an overestimate. Figure 9 shows the actual (generated) diffractive mass together with that

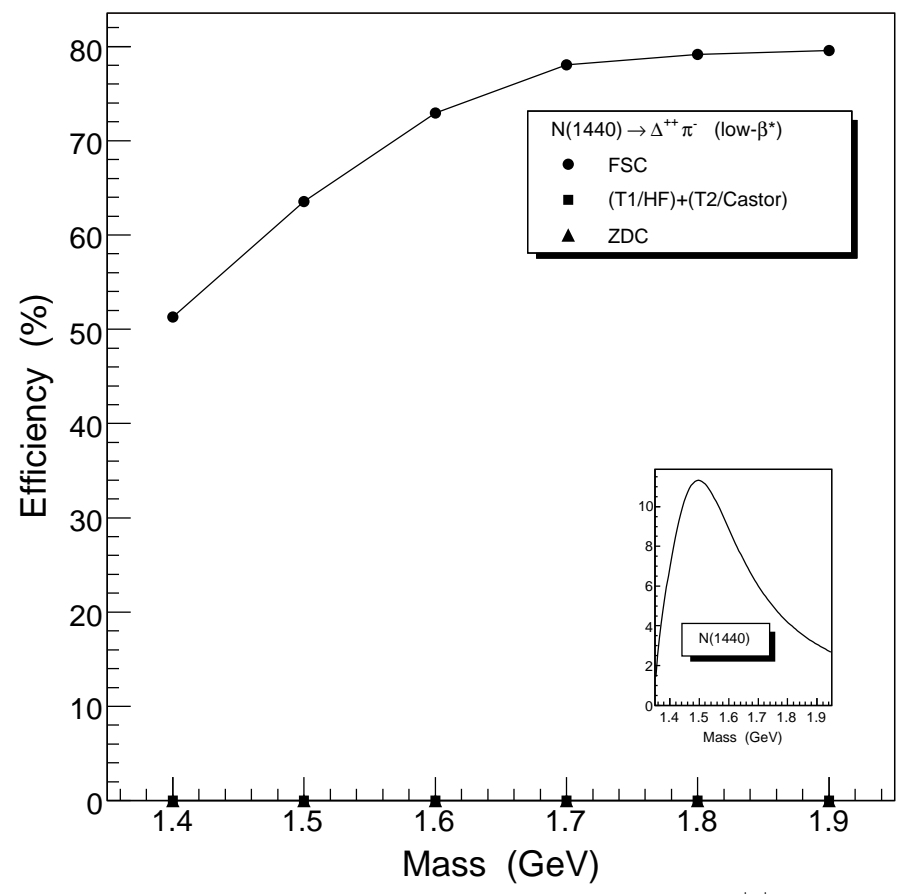

Figure 7. As Fig.5 for $N^{*} \rightarrow \Delta^{++}+\pi^{-}$. 


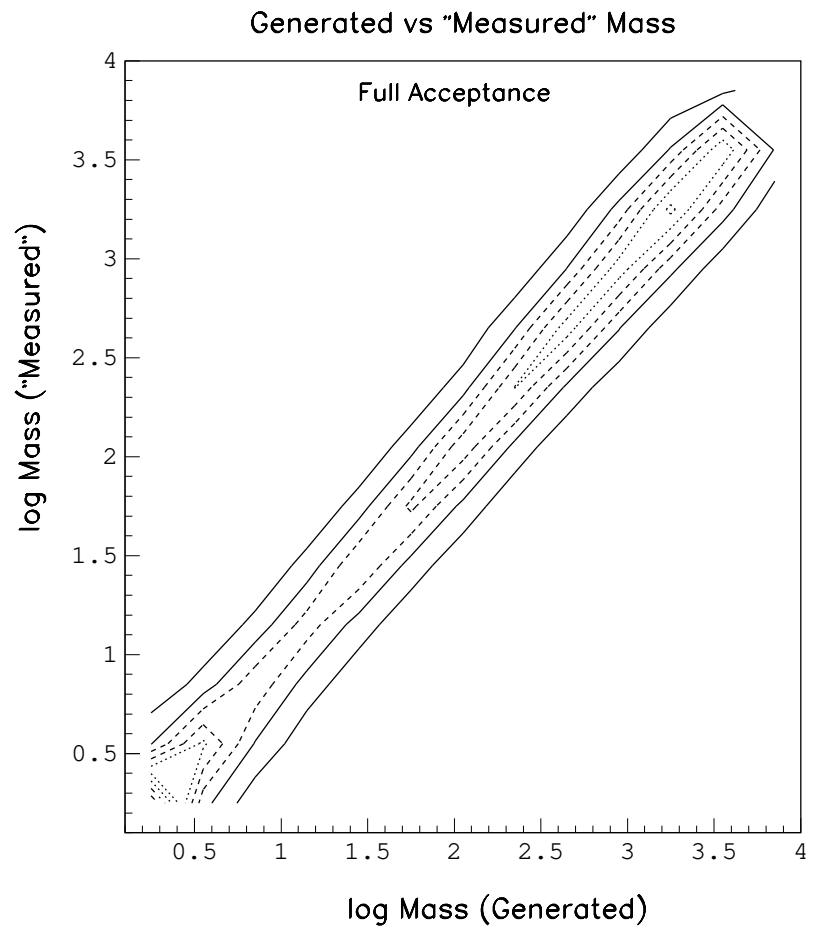

Figure 8. The diffractive mass reconstructed ("measured") from the width of the rapidity gaps vs. the true (generated) mass. The lines show contours of equal density. All forward detectors including the FSC are assumed.

calculated by the above method, for two cases: (a) for full $\eta$ coverage, and (b) for the limited $\eta$ range $|\eta|<4$.7, i.e. the nominal CMS coverage. Clearly the wider the range of rapidity covered, the more accurately the diffractive mass can be determined from the rapidity gap size $\Delta \eta$.

Determination of the diffractive mass on an event-by-event basis from the dependence on $\Delta \eta$ is imprecise for low masses, $M \lesssim 5 \mathrm{GeV} / \mathrm{c}^{2}$. For single diffraction one relies largely on the FSC in this mass range. For central exclusive production the central detectors measure the mass with relatively good precision. The contribution to the total single diffractive cross section in this mass range from the FSC data only is shown by the first solid point and horizontal dashed line in Figure 9, representing the average measurement for the first two bins. Further analysis of the FSC data should improve these results.

The efficiency of the FSC for detecting forward diffractive systems is high. However it is not $100 \%$, and as a result the SDE and CEP studies will contain some background. A subtraction technique can be used to estimate this background and remove it. Data can be taken (a) with, and (b) without the use of the FSC for rapidity gap detection, with T1/HF and T2/CASTOR in veto in both cases. Case (b) includes increased background and characterises the FSC ineficiency. One can also (off-line) measure the content of individual FSC counters, which cover different $\eta$-ranges; this provides more differential tests of the diffractive event simulation. Measuring the various rates, with knowledge of the FSC efficiencies, the background contributions can be estimated and subtracted for different situations (e.g. different $M(X)$ ). Correlations between the counters can be determined 


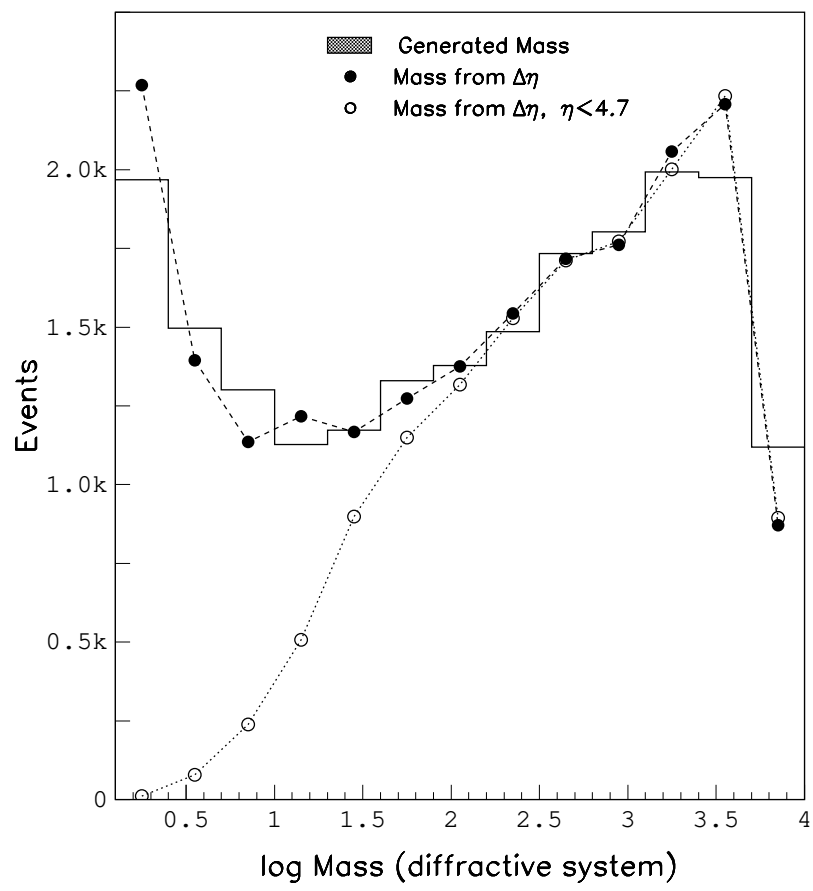

Figure 9. Distribution of the actual (generated) diffractive mass, $\log M(X)$, together with that calculated using the rapidity gap measurement for two cases: (a) full $\eta$ coverage, and (b) for a limited $\eta$ range, $|\eta|<4$.7. The first solid point with the horizontal dashed line indicates the contribution from the FSC data.

and compared with expectations. A valuable check will be the independence of all the measured cross sections on the instantaneous luminosity.

\section{Central exclusive production detection efficiency}

Central exclusive production has two leading protons (not detected without Roman pots or similar devices) adjacent to rapidity gaps of $\gtrsim 4$ units. The $t$-channel 4 -momentum exchanges can be carried by photons, pomerons or (not yet observed) odderons. Therefore the central state $X$ can result from $\gamma \gamma \rightarrow X, \gamma \mathbb{P} \rightarrow X$ or $\mathbb{P} \mathbb{P} \rightarrow X$. As the electromagnetic coupling is much smaller than the strong coupling, the $\gamma \gamma \rightarrow q \bar{q}$ process is an insignificant background to $\mathbb{P} \mathbb{P} \rightarrow$ hadrons, but $\gamma \gamma$ collisions are cleanly observed in exclusive $X=e^{+} e^{-}, \mu^{+} \mu^{-}$[11]. (At high luminosity $\gamma \gamma \rightarrow$ $W^{+} W^{-}$and possible $\tilde{l}^{+} \tilde{l}^{-}$may be observed, in the presence of pile-up and with forward proton measurement.) For low mass exclusive states, e.g. $X=\phi \phi$, note that $p_{T}(X)$ is lower on average from $\gamma \gamma$ exchanges than from IPIP.

Central exclusive production (CEP) was simulated using PHOJET1.1 [18] to generate the central diffractive mass, and PYTHIA to decay the central system into a gluon-gluon dijet. The detection eficiencies for central diffractive events were calculated as functions of the central mass $M(X)$. We required less than five hits in any FSC counter and no tracks in the $\eta$ regions covered by 


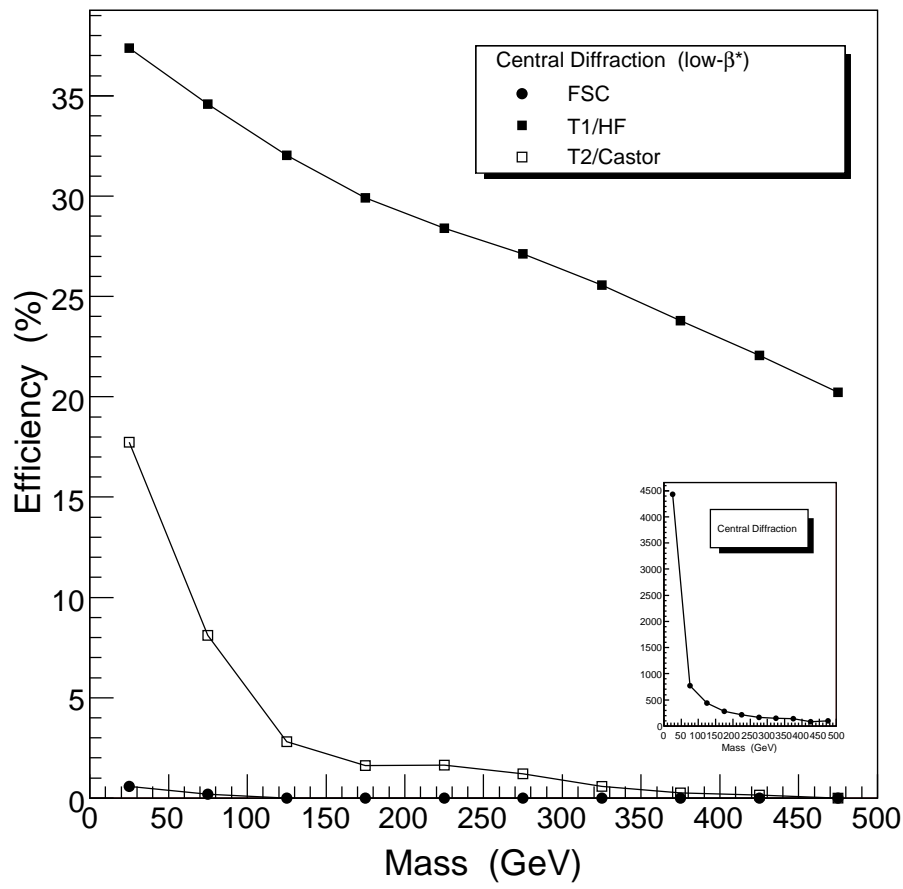

Figure 10. Veto efficiencies for central exclusive diffractive, CED, events simulated by PHOJET1.1/PYTHIA6.2 as a function of the central mass $M(X)$. Separately for the FSC, for T1/HF, and for T2/CASTOR, we call "veto efficiency" the probability that central events will have charged particles in both forward arms.

the $\mathrm{T} 1 / \mathrm{HF}$ and $\mathrm{T} 2 / \mathrm{CASTOR}$ detectors.

For central inclusive diffraction events, we studied the probability of having at least five hits in the FSC (in both arms), and the probability of having at least one track in the T1/HF or T2/CASTOR regions, as a function of the central mass $M(X)$. Requiring an FSC veto is seen to be efficient, and requiring a T2/CASTOR veto is efficient for central masses $M(X) \gtrsim 120 \mathrm{GeV} / \mathrm{c}^{2}$, but requiring a T1/HF veto would reject some $25 \%$ to $35 \%$ of these events, creating a bias. However if one is interested only in the subset of central diffractive production with no particles beyond $|\eta|=$ 3 , the $\mathrm{T} 1 / \mathrm{HF}$ veto would be included.

We have also made simulations of the reactions $p+p \rightarrow p+X+p^{*}$ and $p+p \rightarrow p^{*}+$ $X+p^{*}$, where $p^{*}$ is a forward diffractive system and $X \rightarrow g g$. These reactions are similar to the "quasi-elastic" case where the protons do not dissociate, and the study shows similar results.

As shown in Ref. [10], the cross section of central diffractive production $\frac{d \sigma_{C D}}{d \eta_{1} d \eta_{2}}$ is particularly sensitive to the models of soft diffraction, and these measurements will provide valuable information on the parton content and sizes of various diffractive eigenstates. Measurement of the rapidity gap survival probability, $\hat{S}^{2}$, which determines the diffractive cross sections, is important for understanding strong interaction processes [3, 10]. The present estimates are based on model calculations and $\hat{S}^{2}$ must be experimentally measured. 


\section{Non-diffractive detection efficiency}

The detection efficiency for non-diffractive events was calculated as a function of the charged multiplicity. Requiring at least five hits in any of the FSC counters, and/or at least one track in any region covered by T1/HF or T2/CASTOR, we find the efficiency to be close to $100 \%$, except for very low multiplicity events. The FSC alone have an efficiency for non-diffractive events of about $90 \%$.

\section{Conclusions}

Because of limited forward detector coverage, measurements of single diffractive and central diffractive cross sections in hadron-hadron collisions are more limited at higher $\sqrt{s}$ values than that of the CERN ISR $(\sqrt{s} \leq 63 \mathrm{GeV})$. The published SDE cross sections at the SPS and Tevatron [19] were obtained by extrapolation from data collected in limited $p_{T}$ and $\eta$ regions. At the LHC, diffractive cross sections can be measured with the addition of forward shower counters, FSC, to the present CMS or ATLAS detectors to cover the lowest diffractive masses, below $\sim 10 \mathrm{GeV} / \mathrm{c}^{2}$. With the proposed detector arrangement, valuable new data can be obtained by tagging single and central diffractive processes. The efficiency calculations show that one can use the two-gluon mediated color-singlet interaction as a "gluon collider". The efficiency of the FSC system for detecting rapidity gaps is shown to be adequate for the proposed studies of single- and central-diffraction.

The FSC could also serve as a luminosity monitor by measuring the East-West concidence rate (or equivalently the probability of bunch crossings with no forward particles), as well as monitors of beam conditions.

\section{Acknowledgements}

We thank D.Svododa and R.Hall-Wilton for information on the LHC beam line and discussions.

\section{References}

[1] V.A.Khoze, A.D.Martin and M.G.Ryskin, Prospects for new physics observations in diffractive processes at the LHC and Tevatron, Eur.Phys.J C23 (2002) 311, [hep-ph/0111078 ] .

[2] M.G.Albrow et al. (FP420 R\&D Collaboration), Higgs and new physics with forward protons at the LHC, arXiv:0806.0302 [hep-ex] , to be published in J.Inst.

[3] V.N.Gribov and A.A.Migdal, Properties of the Pomeranchuk pole and the branch cuts related to it at low momentum transfer, Sov. J. Nucl. Phys. 8 (1969) 583; V.N.Gribov and A.A.Migdal, Strong coupling in the Pomeranchuk pole problem, Sov. Phys. JETP 28 (1969) 784, M.G.Ryskin, A.D.Martin and V.A.Khoze, Diffractive processes at the LHC, hep-ph/0506272. 
[4] K.G.Boreskov, A.B.Kaidalov and O.V.Kancheli, Strong interactions at high energies in the Reggeon approach, Phys. Atom. Nuclei, 69 (2006) 1765.

[5] M.G.Ryskin, A.D.Martin, V.A.Khoze and A.G.Shuraev, Soft physics at the LHC, arXiv:0907.1374[hep-ph].

[6] TOTEM, G.Anelli et al., J. Inst. 3 (2008) 508007; also http://totem.web.cern.ch/Totem/

[7] M.G.Albrow et al., Prospects for Diffractive and Forward Physics at the LHC, CERN/LHCC 2006-039/G-124; CMS Note-2007/002; TOTEM Note 06-5 (21st December 2006).

[8] J.W.Lämsä and R.Orava in Diffraction at the LHC, Rio de Janeiro, Feb 2002, R.Orava, Forward physics measurements at the LHC, Proc. of Science, DIFF2006:020 (2006).

[9] M.G.Albrow et al., Forward Shower Counters (FSC) in CMS, a proposal, CMS Internal Note (Dec. 2007).

[10] V.A.Khoze, A.D.Martin and M.G.Ryskin, Early LHC measurements to check predictions for central exclusive production, Eur.Phys.J. C55 (2008) 363 [arXiv:0802.0177]; M.G.Ryskin, A.D.Martin and V.A.Khoze, Soft diffraction at the LHC: a partonic interpretation, Eur. Phys. J. C 54 (2008) 199 [arXiv:0710.2494]; M.G.Ryskin, A.D.Martin and V.A.Khoze, Soft processes at the LHC I:

Multicomponent model, Eur. Phys. J. C 60 (2009) 249; E.Gotsman, E.Levin, U.Maor and J.S.Miller, A QCD motivated model for soft interactions at high energies, arXiv:0805.2799; A.D.Martin, M.G.Ryskin and V.A.Khoze, Forward physics at the LHC, arXiv:0903.2980[hep-ph].

[11] A.Abulencia et al. (CDF Collaboration), Observation of exclusive electron-positron production in hadron-hadron collisions, Phys. Rev. Lett. 98 (2007) 112001; T.Aaltonen et al. (CDF Collaboration), Search for exclusive Z-boson production and observation of high mass $p \bar{p} \rightarrow p+\gamma \gamma+\bar{p} \rightarrow p+l^{+} l^{-}+\bar{p}$ in $p \bar{p}$ collisions at $\sqrt{s}=1.96$ TeV, Phys. Rev. Lett. 102 (2009) 222002; A.Abulencia et al. (CDF Collaboration), Observation of exclusive charmonium production and $\gamma \gamma \rightarrow \mu^{+} \mu^{-}$in $p \bar{p}$ collisions at $\sqrt{s}=1.96 \mathrm{TeV}$, Phys. Rev. Lett. 102, (2007) 242001.

[12] ZDC: O.A.Grachov et al., Performance of the combined zero degree calorimeter for CMS, J. Phys. Conf. Ser.160:012059 (2009); arXiv:0807.0785[nucl.ex].

[13] CASTOR: X.Aslanoglou et al., Performance studies of prototype II for the CASTOR forward calorimeter at the CMS experiment, Eur. Phys. J C52 (2007) 495.

[14] V.A.Khoze, A.D.Martin and M.G.Ryskin, Double-diffractive processes in high-resolution missing-mass experiments at the Tevatron, Eur. Phys. J. C 19 (2001) 477; Erratum ibid C 20 (2001) 599, [arXiv:hep-ph/0011393].

[15] T.Akesson et al., A search for glueballs and a study of double pomeron exchange at the CERN ISR, Nucl. Phys. B 264 (1986) 154.

[16] GEANT: S.Agostinelli et al., Nucl. Inst. Methods 506 (2003) 250; J.Allison et al., IEEE Trans. Nucl. Sci. 53 (2006) 270.

[17] PYTHIA: T.Sjöstrand et al., http://home.thep.lu.se/ torbjorn/Pythia.html

[18] PHOJET: R.Engel and J.Ranft, Hadronic photon-photon interactions at high energies, Phys. Rev. D 54, (1996) 4244.

[19] F.Abe et al., Measurement of p p single diffraction dissociation at $\sqrt{s}=546$ and $1800 \mathrm{GeV}$, Phys. Rev. D 50 (1994) 5535; K.Goulianos and J.Montanha,Factorization and scaling in hadronic diffraction, Phys. Rev. D 59 (1999) 114017. 